\title{
Effects of Sodium Bromide Salt and Temperature on the behaviour of Aqueous Solution of Cetyltrimethylammonium Bromide
}

\author{
Grace A. Cookey* And Charles C. Obunwo \\ Department of Chemistry, Rivers State University of Science and Technology, Nkpolu-Oroworukwo, Port \\ Harcourt, Nigeria
}

\begin{abstract}
The effects of sodium bromide( $\mathrm{NaBr})$ salt and temperature on the aggregation behaviour of cetyltrimethylammonium bromide (CTAB) have been studied using conductivity data.Conductivity was observed to vary increasingly with both temperature and salt concentration. The critical micelle concentration (CMC) of $C T A B$ decreased with increase in the concentration of $\mathrm{NaBr}$ but increased as temperature increased. For example, the CMCs of CTAB in 0.0 and $10.0 \mathrm{mM}$ solutionsof $\mathrm{NaBr}$ at $30{ }^{\circ} \mathrm{C}$ were 1.68 and $1.50 \mathrm{mMrespectively}$ while those of the same concentrations at $35^{\circ} \mathrm{C}$ were 1.84 and 1.63respectively.It wasalso found that both salt and temperature influenced the degree of micelle ionization as well as the free energy of micellization. Results also showedthat the stability of CTAB micelles was enhanced by adding $\mathrm{NaBr}$ molecules. These observations have been discussedin terms of the electrostatic repulsion of charged CTAB head group.
\end{abstract}

Key words:Conductivity, micellization, cetyltrimethylammonium bromide, sodium bromide, temperature and CMC.

\section{Introduction}

A surfactant molecule is a surface-active agent with two distinct moieties- the polar head group and the non-polar alkyl chainpart. Whereas the polar head group may or may not be charged, the non-polar tail group usually comprises of hydrocarbon chains (Bidyut et al, 2009 and Tyowua et al, 2012) though fluorocarbon units also exist. The dual molecular architecture of surfactant molecules distinguishes them from other surface-active agentsin that at low concentrations, they drastically reduce the unusual high surface tension of water through adsorption of the molecules at the air-water interface. Secondly and more significantly, surfactant molecules usually arrange themselves in bulk solutions into aggregates called micelles. Micelle formation is driven by hydrophobic effect but opposed by electrostatic repulsion of ionic head groups (Jiang et al, 2005).

In addition to extensive industrial and household applications of surfactants such as in oil recovery,cosmetics, pharmaceutical, food, detergency, water treatment and micellar solubilisation (Briscoe et al, 2006, Briscoe and Klein, 2007, Sehgal et al, 2008 and Patil et al, 2008),surfactants are also very indispensable in Analytical Chemistry such as in spectrophotometry, electrochemistry and liquid chromatography(Němcováet al, 2009).For instance in spectrophotometry, surfactants influence the absorption spectra of dyes and their complexes with metals. They are also able to influenceacid-base equilibria by altering $\mathrm{pKa}$ values of acid-base media since the $\mathrm{pH}$ of pseudophase micelles is different from that of the aqueous phase(Němcováet al, 2009). In chemical kinetics, theyplay a vital role in influencing rates of chemical reactionsas well as the stability of reaction products.Such effects have been shown to be strongly dependent on the nature of the surfactant head group, the initial counterion present, and the total ionic content of the system (Quina and Chaimovich, 1979).

Surfactants can only function effectively above a certain solution concentration called critical micelle concentration (CMC) i.e., the concentration above which they form aggregates or micelles. It is only above this concentration that significant changes in both physical and performance properties of surfactants take place. The $\mathrm{CMC}$ is influenced by a number of parameters including the nature of surfactant head group and alkyl chain, temperature, pressure, pHand ionic strength. For ionic surfactants, micelle formation is greatly affected by temperature as hydrophobic chain and head group interactions change relatively with temperature (Miller et al, 1990 and Noudehet al, 2007).In other words, inhibition or catalysisby micelles in chemical reactions is a function ofthe surfactant head group. This dependence is greatly influenced by the presence of a common ion from an added salt. Salts bearing the same counterion as that of a surfactant will tend to decrease the fraction of charged sites on micellar headgroup and so reduce the electrostatic interactions on the charged head group resulting in reduced CMC (Bunton C.A. and Cerichelli G., 1980).

The relevance of ionic micelles in the inhibition and/or catalysis of reaction rates in aqueous solutions has been widely studied particularly in view of the analogies drawn between micellar and enzyme catalysis (Katre et al, 2010). The focus has been on the effect of added salts bearing an ion with the counterion of the 
surfactant ion since salts increase the concentration of counterions and thus reduce the number of charged sites on a charged micelle surface.Jones and Jandik, 1991have shown that for inorganic anions, the addition of a cationic surfactant in a concentration above the critical micelle concentration affects the electrophoretic mobility of the analytes and makes it possible to fine-tune the selectivity of the separation. This effect was attributed to the electrostatic interaction of the anions with the cationic micellesand corroborated by Orentaite and Pyell,2011. Although the literature is replete with studies that have been conducted on the thermodynamic properties of surfactants with varying head groups(Noudeh et al, 2007, Bidyut et al, 2009, and Katre et al, 2010)and at different temperatures, there are however, no recorded studies on the effects of temperature and sodium bromidesalt on the thermodynamic properties of CTAB- an important and widely employed surfactant. This study thus attempts to investigate the effects of these parameters on the micellization of CTAB through conductivity measurements.

\section{Materials and Methods}

Cetyltrimethylammonium bromide (98\% purity) and sodium bromidewere purchased from Sigma Aldrich. Deionized water wasprepared in our laboratory. The reagentswere used as received without further purification. Stock aqueous solutions of $\mathrm{CTAB}$ were prepared at concentrations approximately ten times the literature value of its CMC (Azum et al, 2008). Stock solutionsof CTAB were prepared in $0.0 \mathrm{mM}$ and $10.0 \mathrm{mM}$ concentrations of $\mathrm{NaBr}$. Conductivity measurements of all the solutions were carried outwith a JENWAY 4510 conductivity meter at $30,35,40$ and $45{ }^{\circ} \mathrm{C}$. The conductance of a known volume of deionized water was first measured. Subsequently, a precise volume of a stock solution was addedinto the deionized water using an Eppendorf pipette. This addition was repeatedseverally and in each case, the conductivity of the solution was measured.Concentrations of CTABwere calculated from the volumes of the stock solutions used for each measurement.

\section{Results And Discussion}
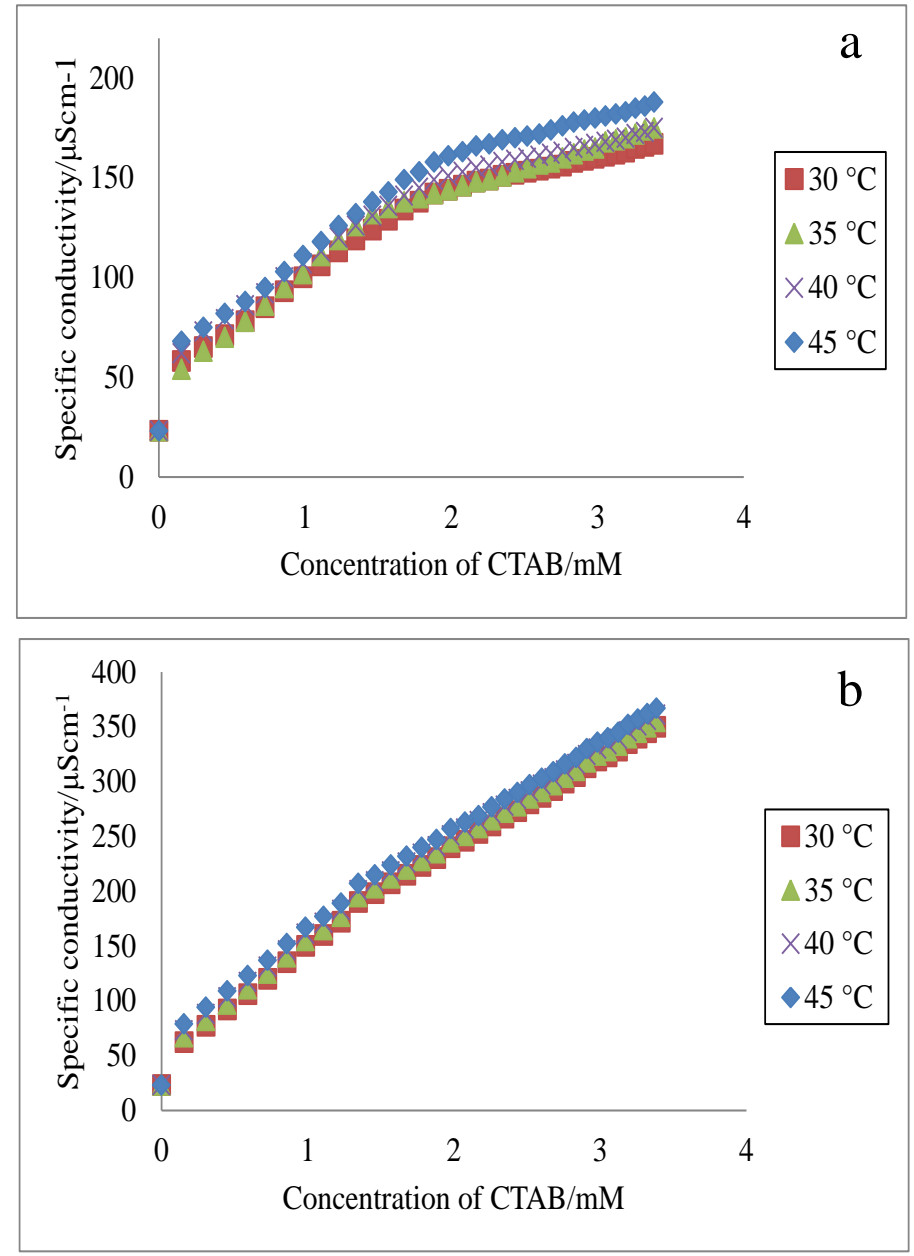

Figure 1: Specific conductivity versus concentration of CTAB, at (a) $0.0 \mathrm{mM}$ and (b), $10.0 \mathrm{mMof} \mathrm{NaBr}$ at different temperatures. 
Figures 1 (a) and (b) show the variation of specific conductivity with CTAB concentration in the presence $(10.0 \mathrm{mM})$ and absence $(0.0 \mathrm{mM})$ of $\mathrm{NaBr}$ at $30,35,40$ and $45^{\circ} \mathrm{C}$. These curves show that the specific conductivity of CTABincreased as the total surfactant concentration increased with a corresponding increase in gradient until aninflectionpoint (the CMC) was observed. Above this point, the specific conductivity still increased with concentration but with a decrease in slope. This abrupt change at the CMC has been reportedin several literatures (Domínguez et al, 1997 and Kroflic et al, 2012)as due to different degrees of ionization of surfactants below and above the CMC. Below the CMC, ionic surfactants behave as strong electrolytes and dissociate completely into their ions. At CMC, aggregates begin to form and mobility of ions is slowed down. Above the CMC, dissociation becomes weaker thus micelles are partially ionized and electrical conductivity then depends on the degree of micelle ionization. Thesecurves also illustrate the dependence of specific conductivity on the concentration of $\mathrm{NaBr}$ salt with specific conductivity increasing asthe concentration of the salt increased.Thisbehaviour may be attributed to increased counterion content in the solution. The cluster of the plots (b) at different temperatures indicates that in the presence of $\mathrm{NaBr}$, temperature has little effect on the specific conductivity of CTAB.

The CMCs of CTAB extrapolated from the inflection points on the specific conductivity versus concentration curves at the various temperaturesandconcentrations of $\mathrm{NaBr}$ salt are illustrated in Figure 2.

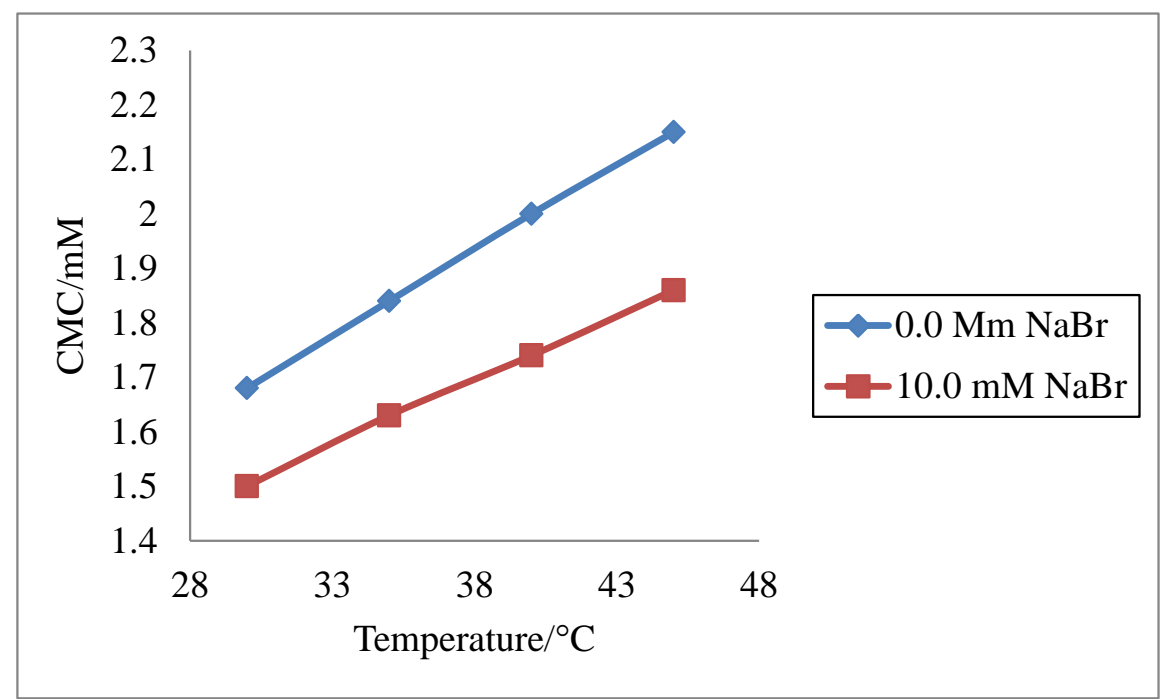

Figure 2: Variation of the critical micelle concentration of CTAB with temperature and concentrations of $\mathrm{NaBr}$.

Figure 2 shows the dependence of the $\mathrm{CMC}$ of $\mathrm{CTAB}$ on both temperature and salt concentration. At a given concentration of $\mathrm{NaBr}$, the $\mathrm{CMC}$ increased with increase in temperature. However, at constant temperature, the $\mathrm{CMC}$ decreased with increasing concentration of $\mathrm{NaBr}$. For instance, the CMC of CTAB in $10.0 \mathrm{mM}$ solution of $\mathrm{NaBr}$ salt at $30{ }^{\circ} \mathrm{C}$ was $1.50 \mathrm{mM}$ whilethat at $35{ }^{\circ} \mathrm{C}$ for same concentration of $\mathrm{NaBr}$ was $1.63 \mathrm{mM}$. At constant temperature of $35{ }^{\circ} \mathrm{C}$, the $\mathrm{CMCs}$ were 1.84 and 1.63 for $0.0 \mathrm{mM}$ and $10.0 \mathrm{mM}$ concentrations of $\mathrm{NaBr}$ respectively. Thermal agitation (as a result of increase in temperature) is expected to increase the electrostatic repulsion of the ionic head group; hence, the chances of micelle formation are reduced leading to higher CMC. Aggregation of surfactant molecules into micelles is enhanced by attractive interactions of the hydrophobic alkyl chains but opposed by the electrostatic repulsion of charged head groups (Domínguez et al, 1997). A decrease in CMC of CTAB with increasing $\mathrm{NaBr}$ concentration may imply that the presence of the salt enhances the formation of CTAB micelles. This is probably because $\mathrm{Br}^{-}$ion of $\mathrm{NaBr}$ increases the surfactant's counterion in solution and thus greatly reduces the number of unbound ionic sites on CTAB micellar head group.Subsequently, the degree of electrostatic repulsion on the charged head groupreduces leading to increased micelle formation.Theseobservations are in agreement with studies (Noudeh et al, 2007)onthe CMC of ionic surfactants at temperatures higher than $25^{\circ} \mathrm{C}$.

Employing the method byKroflicet al. 2012, values of the degree of micelle ionization atthe different temperaturesand in the absence and presence of salt addition were calculated. A linear relationship was observedin both cases (Figure 3).It was also observed that the degree of micelle ionization increased with temperature and with salt concentration. 


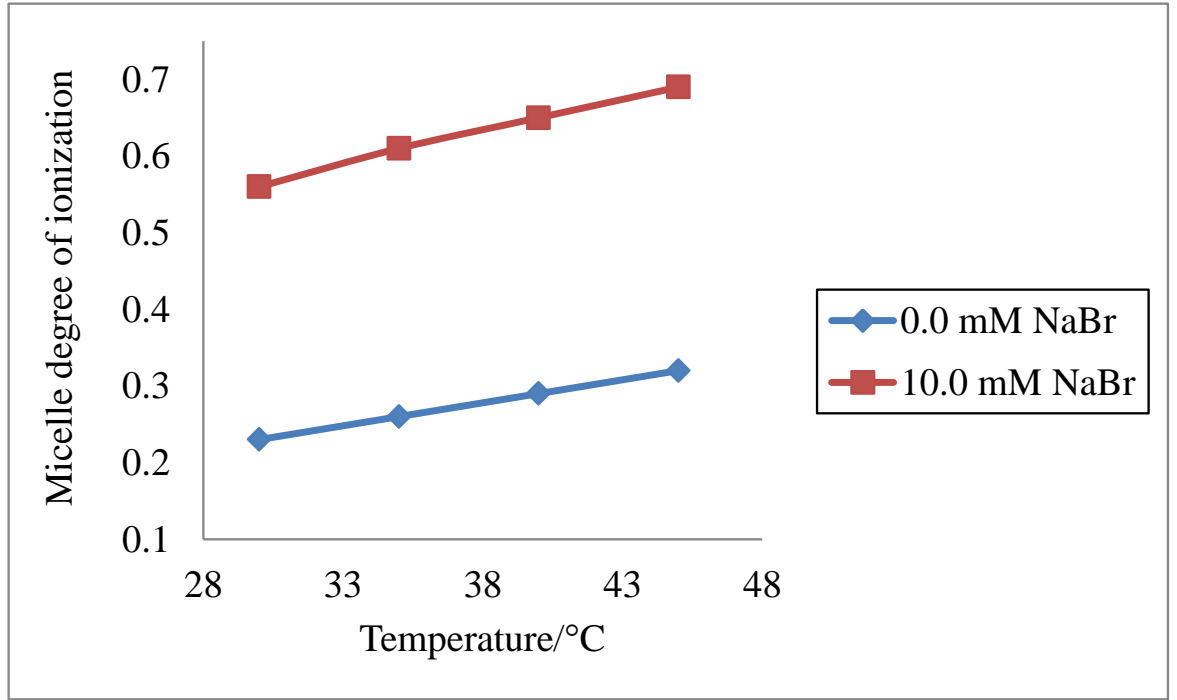

Figure 3: Variation of the degree of micelle ionization of CTAB with temperature in the presence $(10.0 \mathrm{mM})$ and absence $(0.00 \mathrm{mM})$ of $\mathrm{NaBr}$.

This trend agrees with the results obtained by Kroflicet al, 2012on the study of the degree of ionization of alkyltrimethylammonium chlorides at differenttemperatures. They noted that increasing temperature increased the degree of ionization of the surfactant but no distinct influence of added salt was actuallyobserved. The increase in degree of ionization with $\mathrm{NaBr}$ concentration is an indicationthat the ionic strength of CTAB solutions increased with increasing concentration of the saltor the presence of $\mathrm{NaBr}$ increased the number of unbound counterions in CTAB solution. 2007).

The stability of the CTAB micelles formed was evaluated using equation 1(López-Díaz and Velázquez,

$$
\Delta G_{M}=R T \ln X_{C M C}
$$

where $\mathrm{X}_{\mathrm{CMC}}$ is the critical micelle concentration in mole fraction unit, $\mathrm{R}, \Delta \mathrm{G}_{\mathrm{M}}$ and $\mathrm{T}$ are gas constant, free energychange and absolute temperature respectively.

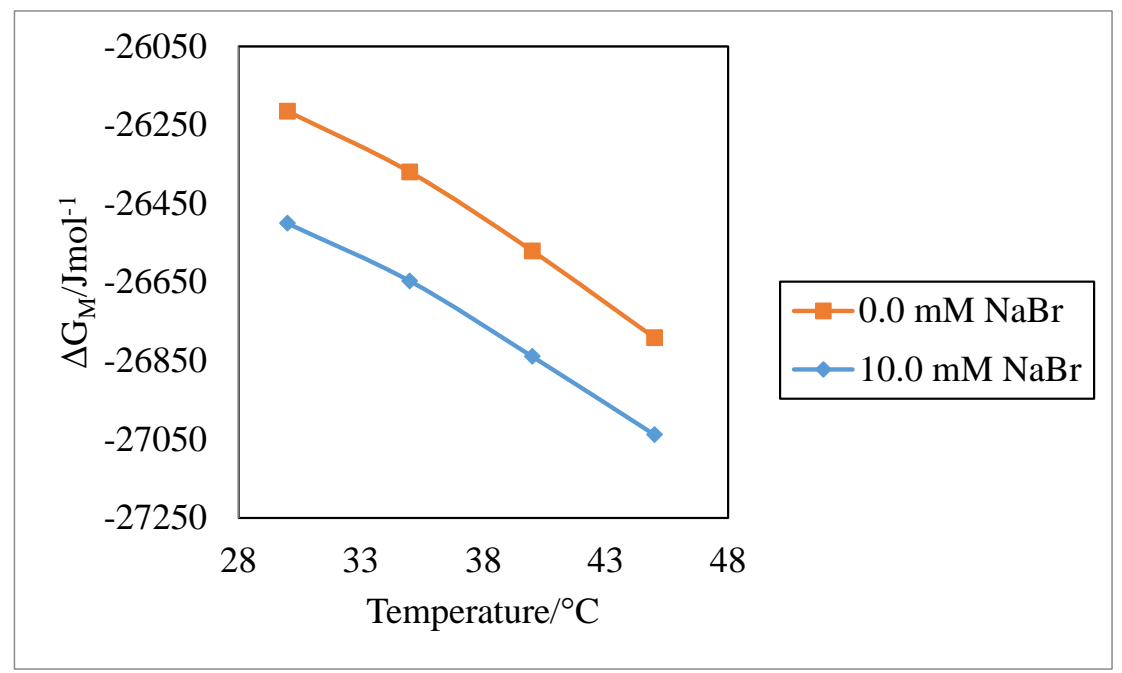

Figure 4: Variation of thechange in free energyof micelle formation $\left(\Delta \mathrm{G}_{M}\right)$ of CTABas a function of temperature and $\mathrm{NaBr}$ concentrations.

The plots in Figure 4 show that at increasing salt concentrations, the magnitudes of the free energy change of micelle formation for CTABincreased with increasing temperature,signifying that CTAB formed more stable micelles in the presence of $\mathrm{NaBr}$ salt. The decrease in $\mathrm{CMC}$ with $\mathrm{NaBr}$ concentration (Figure 2) mightimplythat $\mathrm{NaBr}$ concentration reduced the electrostatic repulsion of charged $\mathrm{CTAB}$ head groupthereby enhancing micelle formation.Consequently, the free energy requiredto transfer each CTAB molecule from the bulk solution into 
the micelle phase might have been reduced in comparison with that required to do same in the absence of the salt. Although the increase in the negative values of the free energy change of micelle formation with temperature was not very clear,it is possible that the sum of the other competing effects may be greater than the reduced repulsion of ionic head group. For instance,dehydration of the ionic head group of a surfactant will normallyincrease with increasing temperaturewhich may result in enhanced micelle formation.

\section{Summary And Conclusion}

Conductivity measurements on the solutions of $\mathrm{CTAB}$ at different temperatures and $\mathrm{NaBr}$ concentrations showed that the $\mathrm{CMC}$ of $\mathrm{CTAB}$ was inversely proportional to $\mathrm{NaBr}$ concentration but directly proportional to temperature. The degree of micelle ionization of CTAB increased with increase in both salt content and temperature. It was also found that the overall stability of CTAB micellesestimated from the change in Gibbs free energy of micelle formationcouldbe enhanced by adding $\mathrm{NaBr}$ molecules. These results have been attributed to the reduction of the repulsive interactions of CTAB ionic head group in the presence of $\mathrm{NaBr}$ salt.

\section{Acknowledgment}

We would like to thank Anna Ochen of the Department of Chemistry, Rivers State University of Science and Technology, Nigeria for helping with some of the data.

\section{References}

[1]. Azum N., Naqvi A.Z., Akram M. and Kabir-ud-Din (2008). Studies of mixed micelle formation between cationic gemini and cationic conventional surfactants. Journalof Colloid and Interface Science, 328, 429-435.

[2]. Bidyut S., Ghosh S.K., Basu A. and Paul K.K. (2009).Micelle catalyzed oxidation of propan-2-ol to acetone by penta-valent vanadium in aqueous acid medium.Journalof molecular Physics, 107, 7, 615-619.

[3]. Briscoe W.H. and KleinJ. (2007).Friction and Adhesion Hysteresis between Surfactant Monolayers in Water. Journalof Adhesion, 83, 705-722.

[4]. Briscoe W.H., Titmuss S., TibergF., Thomas R.K., McGillivray D.J. and Klein J. (2006).Boundary lubrication under water. Nature, 444, 191-194.

[5]. BuntonC.A. and Cerichelli G. (1980).Micellar Effects upon Electron Transfer from Ferrocenes.International Journal of Chemical Kinetics, 12, 519-533.

[6]. Domínguez A., Fernández A., González N., Iglesias E. and Montenegro L. (1997).Determination of critical micelle concentration of some surfactants by three techniques. Journalof Chemical Education, 74, 1227-1231.

[7]. Jiang N., Li P., Wang J., Yan H. and Thomas R.K. (2005).Aggregation of hexadecyltrimethylammonium surfactants with various counterions in aqueous solution. Journalof Colloid and Interface Science, 286, 755-760.

[8]. Jones W.R. and Jandik P. (1991).Controlled changes of selectivity in the separation of ions by capillary electrophoresis. Journal of Chromatography A, 546, 445-458.

[9]. Katre Y.R., Joshi G.K. and Singh A.K. (2010).Kinetic Study of Oxidation of DL-Serine by N-Bromophthalimide in the Presence of Sodium Dodecyl Sulfate. Journalof Dispersion Scienceand Technology, 31, 108-116.

[10]. Kroflic A., SaracB. and RogacM.B. (2012). What Affects the Degree of Micelle Ionization: Conductivity Study of AlkyltrimethylammoniumChlorides. Acta Chimica.Slov.,59, 564-570.

[11]. López-Díaz D. and Velázquez M.M. (2007). Variation of the Critical Micelle Concentration with Surfactant Structure: A Simple Method To Analyze the Role of Attractive-Repulsive Forces on Micellar Association. Journalof Chemical Education, 12, 327-330.

[12]. Miller D.D.,MagidI.J. and Evans D.F. (1990).Fluorescence quenching in double-chained surfactants.2. Experimental results. Journalof Physical Chemistry, 94: 5921-5930.

[13]. NěmcováI., RychlovskýP. and PudilováD. (2009).Effect of Surfactants and Ionic Strength on Dissociation of Hydrochlorides of Phenothiazine Derivatives. Analytical Letters, 42, 646-658.

[14]. Noudeh G.D., Housaindokht M. and Bazzaz B.S.F. (2007).The effect of temperature on thermodynamic Parameters of micellization of some surfactants. Journalof Applied Sciences, 7, 47-52.

[15]. OrentaiteI. and Pyell A.M.U. (2011). Regulation of the retention factor for weak acids in micellar electro kinetic chromatography with cationic surfactant via variation of the chloride concentration.Electrophoresis Journal, 32, 604-613.

[16]. Patil S.R.N., Buchavzov N., Careya E.C. and Stubenrauch C. (2008).Binary mixtures of $\beta$-dodecylmaltoside with cationic and nonionic surfactants: micelle and surface compositions. Soft Matter, 4, 840-848.

[17]. Pawel W. and Maria P. (2005).Miscibility and interaction of dodecyl sulfobetaine with anionic, cationic and nonionic surfactants. Journalof Colloid and Interface Science, 286, 387-391.

[18]. QuinaF.H. and ChaimovichH. (1979).Ion Exchange in Micellar Solutions 1.Conceptual Framework for Ion Exchange in Micellar Solutions. Journal of Physical Chemistry, 83, 1844-1850.

[19]. Sehgal P., Doe H., Wimmer R., Tanaka R. and Kosaka O. (2008).Mixed Monolayer and Micelle Formation of Cationic and Zwitterionic Surfactant of Identical Hydrocarbon Tail in an Aqueous Medium: Interfacial Tension, Fluorescence Probe, Dynamic Light Scattering, and Viscosity Studies. Journalof Dispersion Scienceand Technology, 29, 327-334.

[20]. Tyowua A.T,Yiase S.G. and Wuanna R.A. (2012).Manipulation of concentration-conductivity Data of Sodium Dodecyl Sulphate and Sodium Dodecylbenzene Sulphonate in $\mathrm{KCl}$ Solution in Relation to Micellisation Parameters. Chemical Science Journal, CSJ79, 1-9.

[21]. WeersJ.G.,RathmanJ.F., Axe F.U., CrichlowC.A., FolandL.D., ScheuingD.R., WiersemaR.J. and ZielskeA.G. (1991).Effect of the intramolecular solution properties of betaines and sulfobetaines. Langmuir, 7, 854-867. 